\title{
Severe Pneumonitis with Alveolar Hemorrhage Associated with Herbal Medicines: A Case Report
}

\author{
Miwako Omori, Yoshinobu Saito, Yukiko Miura, Toru Tanaka, \\ Takeru Kashiwada, Kenichiro Atsumi, Hiroki Hayashi, Yuji Minegishi, \\ Kazue Fujita, Arata Azuma, Masahiro Seike and Akihiko Gemma
}

\begin{abstract}
Department of Pulmonary Medicine and Oncology, Graduate School of Medicine, Nippon Medical School, Tokyo, Japan We report a case of pneumonitis with alveolar hemorrhage induced by herbal medicines in a 73-yearold woman who was admitted to our hospital because of dyspnea and an abnormal shadow on a chest radiograph. She had received treatment with numerous drugs, including the herbal medicines Seisinrenshi-in, Chotosan, Rikkunshi-to, and Shakuyakukannzo-to. Chest radiography revealed diffuse ground-glass shadows in both lungs, and bronchoalveolar lavage fluid was progressively hemorrhagic. A culture of the fluid showed no evidence of microorganisms. Moreover, there were no findings suggestive of rheumatic disease or vasculitides. On the basis of this evidence, we suspected drug-induced diffuse alveolar hemorrhage. She discontinued all medicines and started treatment with corticosteroids. Her respiratory condition and chest radiographic findings improved. The timing of administration and rechallenge with other drugs suggested that the herbal medicines were the causative drugs. The primary concern was Seisin-renshi-in, because it contains Ougon (skullcap; a known cause of pneumonitis) and because a drug lymphocyte stimulation test was positive for Seisin-renshi-in. This is the first report indicating that Seisin-renshi-in may cause diffuse alveolar hemorrhage. Diffuse alveolar hemorrhage due to herbal medicines is a rare but emergent disorder. Therefore, treating physicians should be aware that it may be caused by herbal medicines, including Seisin-renshi-in.
\end{abstract}

(J Nippon Med Sch 2019; 86: 296-300)

Key words: drug-induced lung injury, alveolar hemorrhage, herbal medicine, Seisin-renshi-in

\section{Introduction}

Numerous drugs, including herbal medicines, can induce pneumonitis. However, drug-induced pneumonitis is frequently overlooked, and it is often difficult to identify the causative agent. Approximately 150 types of herbal medicines for the treatment of various illnesses and symptoms are currently covered by Japan's health insurance system.

After a report of a death from interstitial pneumonia induced by Sho-saiko-to, physicians were warned of the risk of interstitial pneumonia induced by herbal medicines. Consequently, the package inserts of herbal medicines were revised, and the potential adverse effects associated with these agents were updated. Pneumonitis in- duced by herbal medicines is characterized by various clinical features. Diffuse alveolar hemorrhage (DAH) is an emergent disorder; however, cases of DAH due to herbal medicines are rare. Nevertheless, cases of DAH caused by Makyo-kanseki-to ${ }^{1}$ and Sho-seiryu-to ${ }^{2}$ have been reported. In this report, we describe a case of severe pneumonitis suspected to be associated with DAH caused by herbal medicines.

\section{Case Report}

A 73-year-old woman was admitted to Nippon Medical School Hospital, Tokyo, Japan, because of shortness of breath and cough. Before admission, a few days after onset of dyspnea, productive cough, and anorexia, she had

Correspondence to Miwako Omori, Department of Pulmonary Medicine and Oncology, Graduate School of Medicine, Nippon Medical School, 1-1-5 Sendagi, Bunkyo-ku, Tokyo 113-8603, Japan

E-mail: m-ohs@nms.ac.jp

https://doi.org/10.1272/jnms.JNMS.2019_86-504

Journal Website (https://www.nms.ac.jp/sh/jmanms/) 

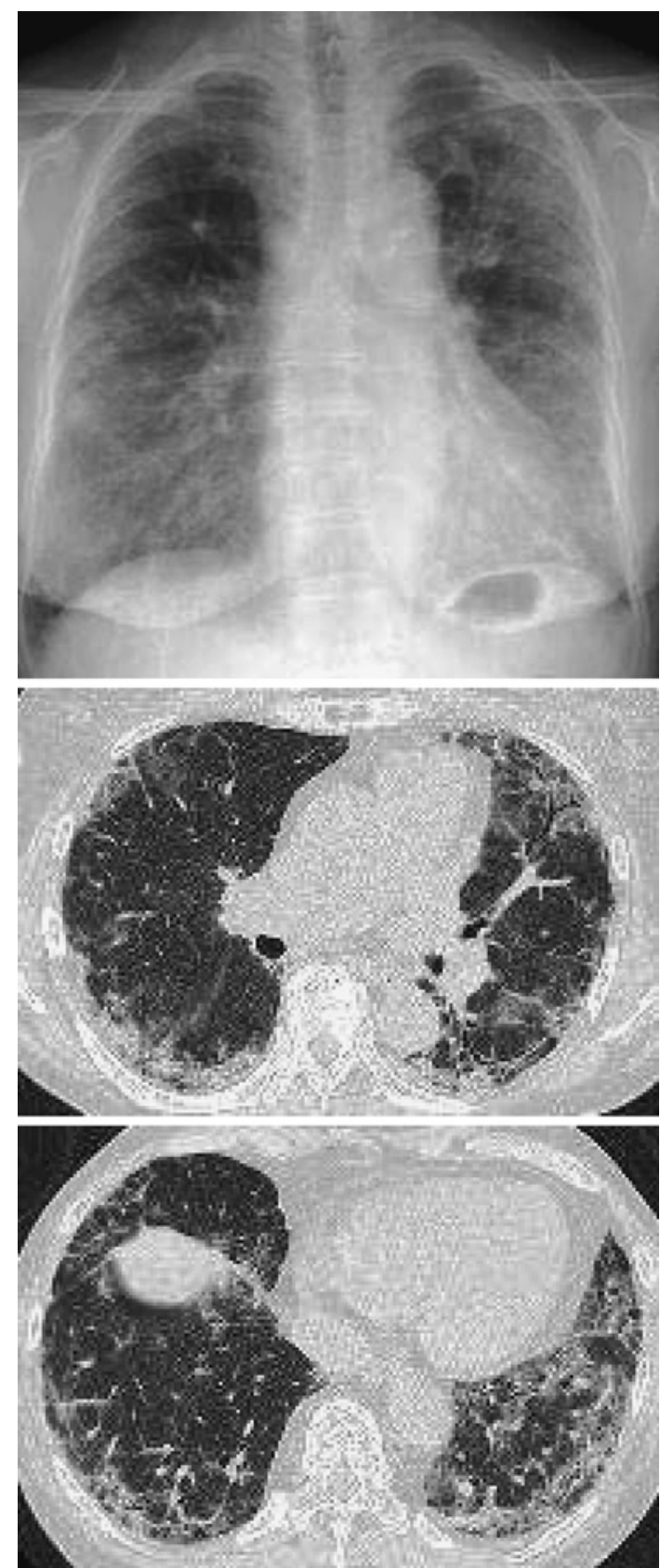

Fig. 1 Chest radiograph and computed tomography image at the time of admission show bilateral infiltrates and ground glass opacities.

a fever of $38^{\circ} \mathrm{C}$. She was examined by a physician at another clinic 4 days later, and a chest radiograph showing a diffuse ground-glass shadow suggested a diagnosis of pneumonitis. She was referred to our hospital on the same day and immediately hospitalized.

The patient was taking multiple herbal medicines for management of dizziness and frequent urination, namely, Shakuyakukannzo-to (for 10 months), Rikkunshi-to (for 6 months), and Seisin-renshi-in and Chotosan (for 2 months). In addition, she had been receiving allopurinol, eicosapentaenoic acid, and alfacalcidol for 10 months,

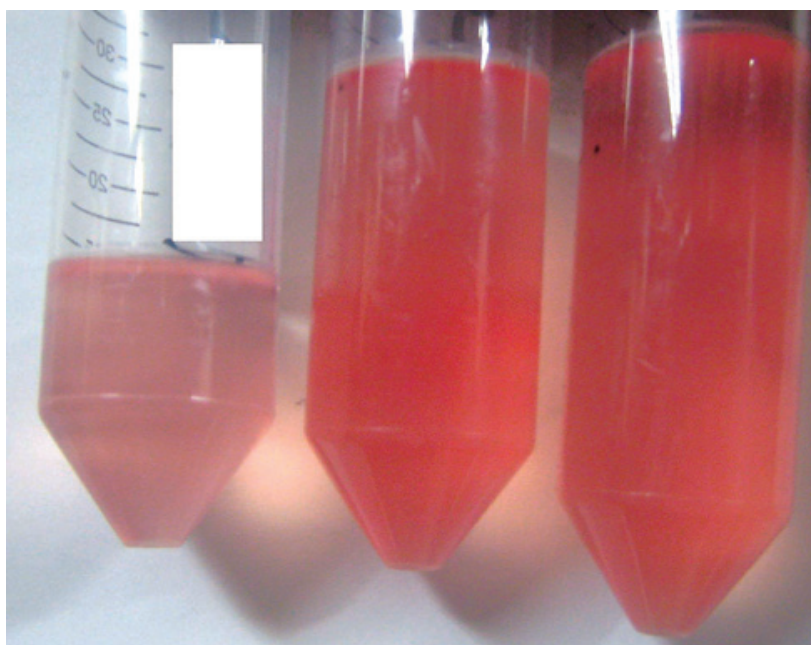

Fig. 2 Photograph of bronchoalveolar lavage shows progressive worsening of hemorrhage.

and nizatidine and zolpidem for 1 year, for treatment of hyperuricemia, dyslipidemia, and osteoporosis.

Physical examination showed a blood pressure of 135/ $71 \mathrm{~mm} \mathrm{Hg}$ and a pulse rate of 82 beats/min. Auscultation revealed inspiratory fine crackles in the bilateral lower lung fields. Cardiac function was normal. Arterial blood gas levels were partial pressure of oxygen $\left(\mathrm{PaO}_{2}\right)$ 66.9 Torr and partial pressure of carbon dioxide $\left(\mathrm{PaCO}_{2}\right)$ 34.7 Torr, and $\mathrm{pH}$ 7.435. Laboratory testing of blood showed significantly elevated levels of white blood cells $(\mathrm{WBC}), \mathrm{C}$-reactive protein (CRP), lactate dehydrogenase (LDH), Krebs von den Lungen-6 (KL-6), and surfactant protein D (SP-D) $(9,100 / \mu \mathrm{L}, 10.21 \mathrm{mg} / \mathrm{dL}, 414 \mathrm{IU} / \mathrm{L}$, $1,947.9 \mathrm{U} / \mathrm{mL}$, and $106.7 \mathrm{ng} / \mathrm{mL}$, respectively). The hemoglobin level decreased from $12.3 \mathrm{~g} / \mathrm{dL}$ before disease onset to $10.7 \mathrm{~g} / \mathrm{dL}$ after onset. Antinuclear antibody, rheumatoid factor, myeloperoxidase antineutrophil cytoplasmic antibody (MPO-ANCA), and proteinase 3 antineutrophil cytoplasmic antibody (PR3-ANCA) were negative. A radiograph and computed tomography (CT) image of the chest showed bilateral infiltrate and groundglass opacities (Fig. 1). Bronchoalveolar lavage (BAL) was performed on the day of admission. Sequential BAL fluid of the left lower lobe was progressively hemorrhagic (Fig. 2). Moreover, differential cell analysis of the lavage revealed 19\% alveolar macrophages, 18\% eosinophils, $8 \%$ neutrophils, and 55\% lymphocytes. The CD4/ CD8 ratio of BAL fluid lymphocytes was 0.2. A culture of the fluid showed no microorganisms. Thus, pneumonitis associated with DAH was diagnosed. Of note, there were no findings suggestive of rheumatic diseases or vasculitides. An adverse drug reaction was suspected to be the 


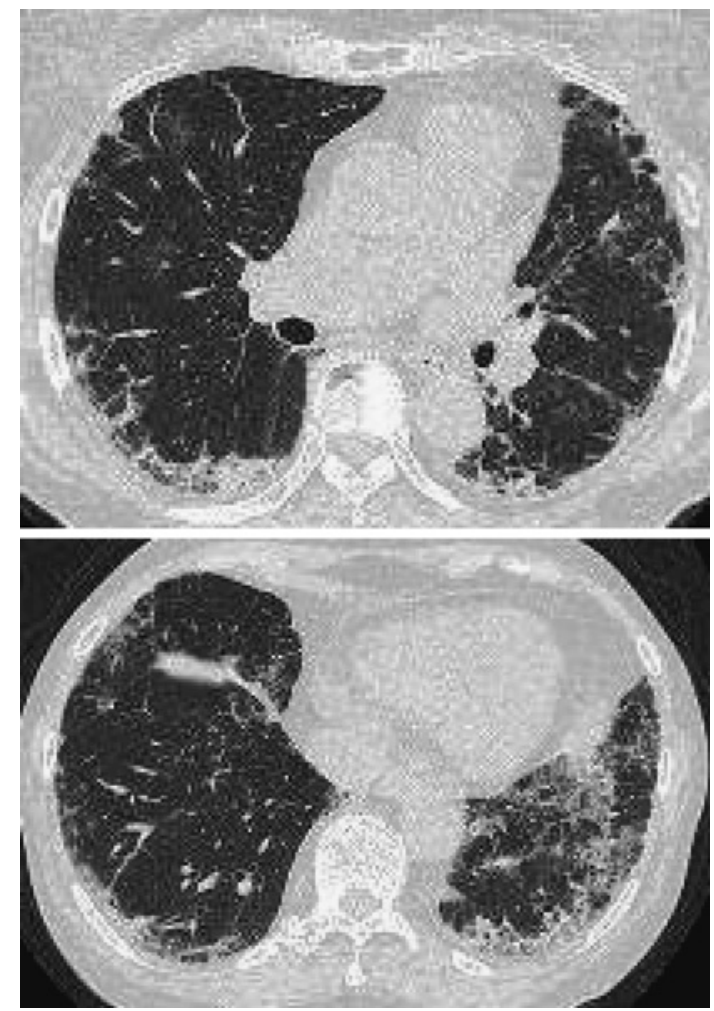

Fig. 3 Chest computed tomography image obtained on day 10 after hospitalization shows improvement in ground glass opacities and infiltrates.

cause of DAH.

After diagnosis, the patient discontinued all medicines, and intravenous methylprednisolone $(1 \mathrm{~g} /$ day) was administered for 3 days. After treatment with methylprednisolone, peripheral oxygen saturation $\left(\mathrm{SpO}_{2}\right)$ and chest radiographic images improved. On day 4 of hospitalization, the steroids were switched to oral prednisolone (1 $\mathrm{mg} / \mathrm{kg}$ ), and the dose was reduced by $10 \mathrm{mg}$ per week. Chest CT on day 10 revealed improvement in groundglass opacities and infiltrates (Fig. 3). Oxygen supplementation was discontinued on day 14 of hospitalization, and the patient was discharged on day 25. Treatment with zolpidem, eicosapentaenoic acid, and allopurinol was restarted after resolution of pneumonitis, but no symptoms developed. Drug lymphocyte stimulation tests (DLST) of Rikkunshi-to, Seisin-renshi-in, Chotosan, and Shakuyakukannzo-to yielded a positive reaction only to Seisin-renshi-in (Table 1).

\section{Discussion}

Terada et al. reported that pneumonitis induced by herbal medicines generally developed within 2 months after the start of treatment ${ }^{3}$. Seisin-renshi-in and Chotosan were administered approximately 2 months before
Table 1 Results of drug lymphocyte stimulation testing (positive index is $\geq 1.8$ )

\begin{tabular}{lc}
\hline \multicolumn{1}{c}{ Herbal medicine } & Stimulation index \\
\hline Chotosan & $1.3(-)$ negative \\
Rikkunshi-to & $1.7(-)$ negative \\
Shakuyakukannzo-to & $0.98(-)$ negative \\
Seisin-renshi-in & $4.8(+)$ positive \\
\hline
\end{tabular}

admission. In contrast, the other agents were administered more than 6 months before onset of symptoms. Moreover, Ougon (skullcap, an ingredient in herbal medicines) was reported to be an important cause of pneumonitis ${ }^{3}$. Among the herbal medicines used by this patient, Seisin-renshi-in was the only agent containing Ougon. In addition, a lymphocyte stimulation test was positive only for Seisin-renshi-in. Furthermore, pneumonitis did not develop after re-administration of any of the drugs that she had been using at the time of admission, other than the herbal medicines. Therefore, we believe that Seisin-renshi-in is the most likely cause of pneumonitis in our patient.

The clinical diagnosis of DAH was based on the presence of bilateral ground-glass opacities on chest CT and the fact that sequential BAL aliquots were progressively hemorrhagic. DAH has multiple causes, including connective tissue diseases, systemic vasculitides, glomerular diseases (i.e., Goodpasture's syndrome), coagulation disorders, and drug toxicity ${ }^{4,5}$. Physical and laboratory examinations of our patient did not yield positive findings for collagen vascular diseases or glomerular diseases, and blood coagulation tests were unremarkable. Therefore, $\mathrm{DAH}$ in our patient was likely attributable to another cause (i.e., drug-induced DAH).

$\mathrm{DAH}$ is a clinicopathological syndrome associated with accumulation of intra-alveolar erythrocytes originating from alveolar capillaries. ${ }^{6}$ Because DAH can be lifethreatening, early diagnosis and identification of its cause are essential. Although hemoptysis is one of the main symptoms of DAH, it is initially absent in up to 33\% of cases, as in the present patient ${ }^{6}$. Other symptoms are nonspecific and include fever, chest pain, cough, and dyspnea. Chest radiographic findings are also nonspecific and consist of a patchy, focal, or diffuse alveolar filling process ${ }^{6}$. A clinical diagnosis of DAH is confirmed when sequential BAL aliquots from the same location are progressively hemorrhagic and the presence of infection can be excluded ${ }^{4}$. Numerous drugs and toxins have been linked to DAH development, including crack cocaine, 
Table 2 Clinical characteristics of interstitial lung disease induced by Seisin-renshi-in

\begin{tabular}{|c|c|c|c|c|c|c|c|c|c|}
\hline Case & $\begin{array}{c}\text { Age } \\
\text { Gender }\end{array}$ & $\begin{array}{c}\text { Duration of } \\
\text { administration }\end{array}$ & $\begin{array}{l}\text { WBC } \\
/ \mathrm{uL}\end{array}$ & $\begin{array}{c}\text { CRP } \\
\mathrm{mg} / \mathrm{dL}\end{array}$ & $\begin{array}{l}\mathrm{LDH} \\
\mathrm{IU} / \mathrm{L}\end{array}$ & $\begin{array}{c}\text { KL-6 } \\
\mathrm{U} / \mathrm{mL}\end{array}$ & DLST & Treatment & Outcome \\
\hline Tagawa [11] & $68 \mathrm{M}$ & 2 weeks & 11,600 & 12.6 & ND & ND & Positive & Drug cessation & Improved \\
\hline Nagato [12] & $83 \mathrm{M}$ & 10 days & 7,250 & 16.88 & 603 & 1,130 & Positive & $\begin{array}{l}\text { Drug cessation } \\
\text { Corticosteroid }\end{array}$ & Expired \\
\hline Nogami [13] & $79 \mathrm{M}$ & 2 months & 4,300 & 0.2 & 289 & 1,744 & ND & Drug cessation & Improved \\
\hline Tsukakoshi [14] & $82 \mathrm{~F}$ & 3 months & 8,600 & 4.16 & 287 & 404 & Negative & Drug cessation & Improved \\
\hline Yokomura [15] & $54 \mathrm{~F}$ & 11 days & 4,620 & 28.8 & 250 & 324 & Negative & Drug cessation & Improved \\
\hline Yokomura [15] & $80 \mathrm{M}$ & 6 months & 6,340 & 1.2 & 281 & 8,660 & ND & Drug cessation & Improved \\
\hline Toyoshima [16] & $62 \mathrm{M}$ & 45 days & 12,100 & 10.2 & 470 & 1,580 & Positive & $\begin{array}{l}\text { Drug cessation } \\
\text { Corticosteroid }\end{array}$ & Improved \\
\hline Haga [17] & $74 \mathrm{M}$ & 2 weeks & 10,100 & 12.77 & 296 & 202 & Positive & $\begin{array}{l}\text { Drug cessation } \\
\text { Corticosteroid }\end{array}$ & Improved \\
\hline Ishimoto [18] & $72 \mathrm{~F}$ & 2 months & 6,500 & 0.16 & 290 & 3,280 & Positive & Drug cessation & Improved \\
\hline Ogata [19] & $70 \mathrm{~F}$ & 20 days & 9,100 & 11.61 & 339 & 612 & Positive & Drug cessation & Improved \\
\hline Present case & $73 \mathrm{~F}$ & 2 months & 9,100 & 10.21 & 414 & $1,947.9$ & Positive & $\begin{array}{l}\text { Drug cessation } \\
\text { Corticosteroid }\end{array}$ & Improved \\
\hline
\end{tabular}

Abbreviations: WBC: white blood cells, CRP: C-reactive protein, LDH: lactate dehydrogenase, KL-6: Krebs von den Lungen-6, DLST: drug lymphocyte stimulation test, ND: no data.

trimellitic anhydride, isocyanates, D-penicillamine, warfarin, nitrofurantoin, propylthiouracil, clopidogrel, fibrinolytics, aspirin, amiodarone, phenytoin, carbamazepine, methotrexate, mitomycin $\mathrm{C}$, radiographic contrast media, and cytotoxic drugs ${ }^{4}$. However, DAH rarely develops after administration of herbal medicines.

General herbal medicines, which are widely prescribed in Japan, consist of multiple herbal ingredients. Currently, the pharmacological mechanisms of herbal medicines are not well understood, and hepatic dysfunction and lung injury are established adverse events caused by herbal medicines. ${ }^{3}$. Although the exact mechanisms of these adverse events are unclear, hypersensitivity is considered a primary cause ${ }^{3}$. In 1989, Tsukiyama et al. reported the first case of pneumonitis induced by Shosaiko-to ${ }^{7}$, which was followed by reports of lung injury (including deaths) induced by Sho-saiko-to ${ }^{8}$. This has become a serious social concern in Japan ${ }^{9}$ and has led to the introduction of guidelines for the safe use of Shosaiko-to ${ }^{10}$.

Several cases of pneumonitis induced by herbal medicines were later reported, notably, 10 cases of interstitial lung disease induced by Seisin-renshi-in ${ }^{11-19}$ (Table 2). The interval to disease onset in these reports varied from 10 days to 6 months. Moreover, elevations in white blood cell count (five cases), eosinophil count (four cases), KL-6 (six cases), CRP (eight cases), LDH (five cases), and hepatic dysfunction (five cases) were observed. Eight patients underwent DLST. Positive reactions to Seisinrenshi-in were observed in five cases, and a suspected positive reaction was observed in one patient ${ }^{12}$. Among the five positive cases, the test result was negative during corticosteroid therapy but became positive after the start of corticosteroid therapy in a case reported by Haga et al. ${ }^{14}$. Nine cases progressed mildly and improved after cessation of drugs and/or after corticosteroid therapy. In 2013, Nagato et al. ${ }^{12}$ reported diffuse alveolar damage induced by treatment with Seisin-renshi-in. In addition, Toyoshima et al. reported lymphocytic infiltration of alveolar septa, desquamative alveolar lining cells, and fibrinous exudate in alveolar spaces ${ }^{16}$. Tagawa et al. ${ }^{11}$ reported hypertrophy of alveolar epithelium and interstitial fibrosis.

This is the first reported case of alveolar hemorrhage induced by treatment with Seisin-renshi-in.

Kondo reported that, in Japan, the DLST positive rate was $66.9 \%$ and $67.6 \%$ for drug-induced lung disease (DILD) caused by medicines and herbal medicines, respectively $^{20}$. Furthermore, Enomoto et al. reported that the DLST positive rate for cases of DILD caused by Japanese herbal medicines was $56 \%{ }^{21}$. DLST is a noninvasive examination tool for auxiliary diagnosis of DILD. However, it is limited by the high risk of false-positive results and the weak correlation between positive DLST results and drugs suspected to induce pneumonitis ${ }^{32,223}$. A previously reported problem is that, although lymphocyte reactions differ, the same reference value is used for all drugs $^{18}$. In addition, treating physicians should take into account that corticosteroid therapy may induce falsenegative results in $\mathrm{DLST}^{17}$. Therefore, future studies 
should attempt to determine the usefulness of DLST in DILD diagnosis.

Herbal medicines can cause adverse effects, and deaths from pneumonitis caused by herbal medicines have been reported. The risks of adverse drug reactions (including DILD) should be explained to patients, and they should be advised to consult their physicians if symptoms (e.g., cough, fever, and dyspnea) develop after starting treatment with herbal medicines. The clinical history and medication records of patients presenting with pneumonitis should be evaluated, especially if they are treated with multiple agents. Reaching a definitive diagnosis of DILD is often difficult. However, prompt action, including discontinuation of treatment, is necessary in patients at high risk of developing DILD.

\section{Conclusion}

We reported a case of severe pneumonitis with DAH in a patient receiving treatment with four herbal medicines. Seisin-renshi-in was the most likely cause of pneumonitis. Given the wide use of herbal medicines, treating physicians should recognize these agents as a potential cause of pneumonitis with DAH.

Conflict of Interest: The authors have declared no conflict of interest.

\section{References}

1. Iida $\mathrm{Y}$, Takano $\mathrm{Y}$, Ishiwatari $\mathrm{Y}$, Yoshida A, Shimizu T, Ito R, Hattori T, Takahashi N, Hashimoto S: Diffuse alveolar Hemorrhage Associated with Makyo-kanseki-to Administration. Intern Med 2016; 55: 3321-3323.

2. Tsuchiya K, Toyoshima M, Suda T: Pneumonitis with Alveolar Hemorrhage Induced by Sho-seiryu-to. Intern Med 2017; 56: 2623-2626.

3. Terada M, Kitazawa H, Kawakami J, Adachi I: Pharmacoepidemiology of Interstitial Pneumonia and Liver Dysfunction Associated with Kampo Medicine. Jpn J Pharm Health Care Sci 2002; 28: 425-434 (in Japanese, Abstract in English).

4. Newsome B, Morales J: Diffuse Alveolar Hemorrhage. Southern Medical Journal 2011; 104: 269-275.

5. Park MS: Diffuse Alveolar Hemorrhage. Tuberc Respir Dis 2013; 74: 151-162.

6. Lara A, Schwarz M: Diffuse Alveolar Hemorrhage. CHEST 2010; 137: 1164-1171.

7. Tsukiyama K, Tasaka Y, Nakajima M, Hino J, Nakahama T, Okimoto J, Yagi S, Soejima R: A case of Pneumonitis due to Sho-saiko-to. Nippon Kyobu Shikkan Gakkai Zasshi 1989; 27: 1556 (in Japanese, Abstract in English).

8. Sato A, Toyoshima M, Kondo A, Ohta K, Sato H, Ohsumi A: Pneumonitis induced by the herbal medicine Shosaiko-to in Japan. Nihon Kyobu Shikkan Gakkai Zasshi 1997; 35: 391-395 (in Japanese, Abstract in English).

9. Tomioka H, Hashimoto K, Ohnishi H, Fujiyama R, Sakurai T, Tada K, Sakamoto H, Iwasaki H: An autopsy case of interstitial pneumonia probably induced by Sho- saiko-to. Nihon Kokyuki Gakkai Zasshi 1998; 37: 10131018 (in Japanese, Abstract in English).

10. Suzuki H, Kumada H, Sato A, Shiraki K, Honma Y, Kogure T, Terasawa K: Guidelines of Sho-saiko-to/ XiaoChaohu-Tang treatment in patients with chronic hepatitis C. Journal of Traditional Medicines 2000; 17: 95-100 (in Japanese, Abstract in English).

11. Tagawa T, Kimura K, Sekiguchi N, Watanabe H, Hayashi M: A case of interstitial pneumonitis induced by Chinese Medicine, Sei-sin-rensi-inn. Kokyu 1999; 18: 662-666 (in Japanese, Abstract in English).

12. Nagato T, Iseki M, Morikawa A: A Case of Drug-induced Lung injury due to Seisin-rensi-in. Jpn J Chest Dis 2013; 72: 1021-1028 (in Japanese, Abstract in English).

13. Nogami $T$, Jo $M$, Inoue $H$, Fujimoto $M$, Hikiami $H$, Shimada Y: Syusekisantoruterozin oyobi seishinrenshiin nite kannshituseihaien wo hannpukushitaitirei [A case of recurring interstitial pneumonia by tartaric acid and seisinrenshiin]. Journal of Kampo Medicine 2013; 60: 289295 (in Japanese).

14. Tsukakoshi H: A case of pneumonitis caused by Seishinrenshiin, Chinese herbal medicine. The Allergy in Practice 2010; 30: 646-649 (in Japanese, Title in English).

15. Yokomura K, Matsushima S, Oyama $Y$, Kusagaya $H$, Yasui H, Matsui T, Matsuda H, Nakano Y, Suda T, Chida $\mathrm{K}$ : Two cases of pneumonitis caused by seishinrenshiin Chinese herbal medicine. Allergy 2009; 58: 1441-1446 (in Japanese, Abstract in English).

16. Toyoshima M, Chida K, Suda T, Harada M: A case of pneumonitis caused by Seisin-renshi-in, herbal medicine. The Journal of the Japanese Respiratory Society 2008; 46: 31-34 (in Japanese, Abstract in English).

17. Haga T, Fukuoka M, Morita M, Cho K: A Case of Drug Induced Pneumonia Caused by Seishinrenshiin that was Examined with the Sequential Lymphocyte Stimulation Test. Respiration and Circulation 2015; 63: 183-186 (in Japanese, Abstract in English).

18. Ishimoto $\mathrm{H}$, Yatera $\mathrm{K}$, Hanaka $\mathrm{T}$, Oda $\mathrm{K}$, Kawamoto $\mathrm{S}$, Mukae H: A Case of Drug-induced Lung Disease Caused by Seishin-renshi-inn Extract. The Japanese Journal of Chest Diseases 2016; 75: 428-432 (in Japanese, Abstract in English).

19. Ogata K, Satomura T, Morooka M: A Case of Druginduced Pulmonary Disease Considered to be Caused by Seishinrenshiin with Worsening Infiltrative Shadows after Discontinuation of the Drug. The Japanese Journal of Chest Diseases 2016; 75: 1468-1472 (in Japanese, Abstract in English).

20. Kondo A: Drug-induced pneumonitis. Kekkaku 1999; 74: 33-41 (in Japanese, Abstract in English).

21. Enomoto Y, Nakamura Y, Enomoto N, Fujisawa T, Inui N, Suda T: Japanese herbal medicine-induced pneumonitis: A review of 73 patients. Respiratory Investigation 2017; 55: $138-144$.

22. Komiya $\mathrm{K}$, Ishii $\mathrm{H}$, Ohama $\mathrm{M}$, Uchida $\mathrm{M}$, Tsubone $\mathrm{T}$, Iwashita T, Miyajima H, Okabe E, Matsumoto T, Matsumoto B, Kadota J: Sai-rei-to-induced Lung Injury: A Case Report and Brief Review of the Literature. Intern Med 2012; 51: 3421-3425.

23. Mantani N, Kogure T, Tamura J, Shimada Y, Terasawa K: Lymphocyte Transformation Test for Medicinal Herbs yields False-Positive Results for First-Visit Patients. CLIN DIAGN LAB IMMUNOL 2003; 10: 479-480.

(Received, November 19, 2018)

(Accepted,

April 17, 2019)

(J-STAGE Advance Publication,

May 17, 2019) 